Fisioter Bras 220;21(2):149-63

https://doi.org/10.33233/fb.v21i2.2499

\title{
ARTIGO ORIGINAL \\ Efeitos de um programa fisioterapêutico com terapia por tarefas orientadas e treino de marcha para trás na locomoção de pacientes após acidente vascular encefálico: série de casos \\ Effect of a physical therapy program with task-oriented approach and backward gait training in the locomotion after stroke: case series
}

Suzanne Guimarães Machado, Ft. *, Jaíne Rosalva de Aguiar, Ft.. Ricardo de Oliveira Santana, Ft. ${ }^{*}$, Raphaela Schiassi Hernandes, TO.**, Sheila Schneiberg, Ft. ${ }^{* \star}$

${ }^{*}$ Fisioterapeutas egressos do Departamento de Fisioterapia, Universidade Federal de Sergipe, Campus Lagarto, ${ }^{*}$ Docente, Departamento de Terapia Ocupacional, Universidade Federal de Sergipe, Campus Lagarto, ${ }^{\star * \star D}$ Docente, Departamento de Fisioterapia, Universidade Federal de Sergipe, Campus Lagarto

Recebido 31 de agosto de 2018; aceito 15 de fevereiro de 2020.

Correspondência: Sheila Schneiberg, Departamento de Fisioterapia, Universidade Federal de Sergipe, Campus Lagarto, Av. Governador Marcelo Déda, s/n, Centro 49400-00 Lagarto SE

Sheila Schneiberg: sheilaschneiberg@gmail.com

Suzanne Guimarães Machado: suzanne.machado1@hotmail.com

Jaíne Rosalva de Aguiar: jaineaguiar-2@hotmail.com

Gabriel Pacheco Bispo: gabrielpbispo91@gmail.com

Ricardo Oliveira Santana: ricardoo437@gmail.com

Raphaela Schiassi Hernandes: rapha_to@hotmail.com

\section{Resumo}

Introdução: Pacientes após Acidente Vascular Encefálico (AVE) costumam ter alteração da marcha, apresentando velocidade lenta, baixa resistência a longas distâncias, e limitação da locomoção independente nas ruas. A recuperação dessa marcha comunitária é para esses indivíduos, um dos principais objetivos para a manutenção de uma vida ativa. Objetivo: Investigar a eficácia de uma intervenção fisioterapêutica com o método de tarefas orientadas e com o treino de marcha para trás sobre a locomoção de pacientes hemiparéticos após AVE. Métodos: Tratase de um estudo de análise de efeito terapêutico do tipo série de casos, no qual o sujeito é seu próprio controle, e são realizadas avaliações múltiplas para cada sujeito nas fases A1- B - A2. $\mathrm{Na}$ fase $\mathrm{A} 1$ são feitas duas avaliações antes do tratamento, gerando uma linha de base (baseline) que por ter duas medidas inclui a variação normal do sujeito, na fase $B$ também são feitas duas avaliações, mas, durante e imediatamente após o tratamento, para obter as mudanças relacionadas ao mesmo e na fase A2 são feitas duas avaliações após duas semanas e um mês sem tratamento, para avaliar retenção do tratamento. Este estudo foi realizado com indivíduos com sequelas de AVE deambuladores comunitários (capazes de andar na rua, com ou sem auxílio de órteses). Os constructos utilizados para avaliar o efeito da terapia nos participantes foram: equilíbrio avançado, marcha comunitária com capacidade de modificar marcha as tarefas funcionais, equilíbrio e mobilidade, que foram investigados respectivamente com os instrumentos Fullerton Advanced Balance (FAB), Índice de Marcha Dinâmica (DGI) e Timed Up Go (TUG). Dois métodos estatísticos foram utilizados: 1) O método visual da banda formada pela média $\mathrm{e}$ por dois desvios padrão calculados com os resultados das avaliações feitas na baseline, formando uma banda cujos pontos que caírem fora da banda tem uma significância $p<0.05$; e 2) O cálculo do tamanho do efeito da terapia com o método de Delta de Glass. Resultados: Participaram do estudo quatro voluntários com sequelas após AVE. Após aplicação do protocolo de intervenção com tarefas orientadas e marcha para trás, observou-se melhora do equilíbrio e mobilidade, assim como da locomoção e da capacidade de modificação da marcha às atividades funcionais, isso pode ser observado especialmente no TUG em que todos os participantes diminuiram o tempo, após o tratamento em aproximadamente 3 a 20 segundos, $p<0,05$. Os tamanhos do efeito após terapia foram grandes a enormes $>0,80$ e 1,30 para todos os participantes em dois dos três desfechos primários. Conclusão: A associação do treino por 
tarefas orientadas com o treino de marcha para trás dentro do processo de reeducação funcional de pacientes após AVE surte efeitos positivos sobre a locomoção.

Palavras-chave: acidente vascular cerebral, locomoção, marcha, tarefas orientadas, série de casos.

\begin{abstract}
Introduction: Patients after stroke usually have gait dysfunctions, presenting slow speed, low resistance over long walking distances, and limitation of independent walking outdoors. The recovery of community walking is for those individuals, one of the main goal necessary to keep an active life. Objective: To investigate the effectiveness of a physical therapy intervention with the task-oriented method associated with backward gait training on the locomotion of hemiparetic patients after stroke. Methods: This study is a case series, to analyze therapeutic effect, in which the subject is his own control, and multiple evaluations are performed for each subject in phases A1 - B - A2. In phase A1 two pre-treatment assessments are performed, generating a baseline that includes the normal variation of the subject. In phase B two assessments are also made, but during and immediately after treatment to obtain changes related to the treatment effect and, in phase A2, two assessments are made, after two weeks and one month without treatment, phase A2 is to assess retention of treatment. This study was conducted with community-walking strokes survivors (capable of walking on the street, with or without orthoses). The constructs used to assess the effect of therapy on participants were: advanced balance, community gait with ability to change functional tasks, speed and mobility, which were investigated with the Fullerton Advanced Balance (FAB) instruments, Dynamic Gait Index (DGI) and Timed Up Go (TUG). Two statistical methods were used: 1) The visual method of the band formed by the mean and by two standard deviations calculated from the results of the baseline evaluations, forming a band where the points that fall outside the band have a significance of $p<0.05$ and 2) The size calculation of the effect of therapy with the Glass Delta method. Results: Four volunteers with stroke sequelae participated in the study. After applying the intervention protocol with task-oriented approach and backward gait training, it was observed an improvement in balance and mobility, as well as locomotion and gait modification ability to functional activities, that was observed especially in the TUG where all participants decreased the time after treatment in approximately 3 to 20 seconds, $p<0.05$. Post-therapy effect sizes were large to huge $>0.80$ and 1.30 for all participants in two of the three primary outcomes. Conclusion: The association of task-oriented training with backward gait training within the functional rehabilitation process of patients after stroke has positive effects on locomotion.
\end{abstract}

Keywords: stroke, locomotion, gait, task oriented, case series.

Introdução

O Acidente Vascular Encefálico (AVE) pode ser definido como uma disfunção neurológica, em determinada área encefálica, que tem como etiologia uma lesão vascular, podendo ser do tipo isquêmica ou hemorrágica [1]. É considerado uma das principais causas de incapacidade da atualidade, já que $80 \%$ das pessoas afetadas tornam-se dependentes de auxílio para no mínimo uma atividade funcional [2]. O AVE é um relevante problema de saúde pública, com uma taxa de alta letalidade e quantidade significativa de sobreviventes com dependência funcional, pois, a cada ano, 15 milhões de pessoas no mundo são acometidas [3]. Deste quantitativo, cinco milhões de pessoas vão à óbito enquanto outros cinco milhões sobrevivem com incapacidades, o que gera graves consequências nos contextos pessoal, familiar e de participação comunitária [4]. No Brasil, embora tenha havido declínio das taxas de mortalidade, - AVE representa a primeira causa de morte e incapacidade em todo o país, o que repercute negativamente nos aspectos econômico e social nacional [3]. Dados advindos de estudo prospectivo nacional evidenciaram incidência anual de 108 casos por 100 mil habitantes, taxa de fatalidade aos trinta dias de 18,5\%, e, aos 12 meses, de 30,9\%, e índice de recorrência após um primeiro episódio de AVE de 15,9\% [5].

Após a lesão vascular, cerca de $40 \%$ dos indivíduos não são capazes de deambular de forma independente na comunidade, e a dependência persiste mesmo após uma melhora global significativa com a reabilitação [6-11]. A marcha de pacientes com hemiparesia costuma ter como características marcantes a menor amplitude dos movimentos articulares, velocidade reduzida, assimetria na transferência do peso corporal, aumento da fase de balanço, instabilidade na fase de apoio, elevado gasto energético, déficit no ritmo e lentidão nos mecanismos adaptativos posturais diante de distratores do ambiente $[6-8,11]$. 
Um aspecto importante na marcha hemiparética é a assimetria na descarga de peso durante o apoio unipodal [12]. A natureza assimétrica da locomoção de hemiparéticos é resultado de uma atividade muscular anormal, controle deficiente dos mecanismos posturais e prejuízo sensorial $[12,13]$. Mesmo com a recuperação da capacidade de andar, a maioria dos pacientes permanece com uma marcha de velocidade lenta e baixa resistência a longas distâncias, e sua locomoção independente fora de casa e na sociedade são limitados [14-16].

Dentre as possibilidades atuais de intervenção fisioterapêutica na marcha hemiparética, destaca-se o treino por meio de tarefas orientadas. É baseado nas teorias atuais de controle motor e aprendizagem motora [17,18], difere de práticas de treino fisioterapêutico tradicionais em que a força muscular é treinada de forma isolada e fora da tarefa funcional do músculo a ser fortalecido ou ainda de tratamentos que focam nos componentes patológicos da marcha (ex. amplitude de tornozelo) de maneira isolada, fora do contexto funcional e tarefas durante as fases da marcha. No treino por tarefas orientadas, a força, amplitude, destreza e coordenação do movimento são treinados em um contexto funcional, ou seja, se o objetivo é melhorar a marcha, os componentes disfuncionais da mesma são tratados durante a marcha e suas demandas funcionais exigidas em cada fase, utilizando práticas e feedbacks variados [17-19].

Alguns estudos demonstraram resultados com melhora da velocidade e padrão da marcha em pacientes com AVE submetidos ao tratamento com uso de tarefas orientadas. Essa melhora foi acompanhada pelo aumento do desempenho nas atividades de vida diária, aumento do equilíbrio e força muscular dos membros inferiores, e redução do número de quedas neste público [20-23].

Apesar de que na prática clínica da fisioterapia neurofuncional o andar para trás ter sido discutido e comumente empregado desde o início do ano 2000 [24], em geral, no treino fisioterapêutico neurofuncional de marchas hemiparéticas, a marcha normal ou para frente é mais comumente utilizada. Apenas recentemente, alguns estudos têm pesquisado o efeito da marcha para trás em pacientes com sequela de AVE [25-31]. O Backward walking ou marcha para trás possue diferentes solicitações funcionais e combinações musculares quando comparado a marcha para frente [26], principalmente por associar a extensão do quadril com a flexão do joelho, e o contato inicial ser com o ante-pé. Dessa maneira, o treino de marcha para trás pode melhorar os componentes da marcha de indivíduos hemiparéticos, em que há presença de sinergia patológica nas extremidades inferiores. Dados de um estudo mostrou que o caminhar para trás e o andar para a frente diferiam em suas características cinésio - fisiológicas e que os traços temporais e espaciais do andar para trás poderiam elevar a frequência cardíaca e a resistência à caminhada [27,28]. A literatura indica que o treino com marcha para trás [29-31] pode oferecer alguns benefícios além daqueles experimentados através do caminhar para a frente, como gerar mais atividade muscular em proporção ao esforço e aumento da velocidade e desempenho geral da marcha.

A combinação do método por tarefas orientadas com o treino utilizando a marcha para trás parece ser uma proposta promissora na recuperação da marcha comunitária de pacientes após AVE. Contudo, não há atualmente evidências científicas acerca da reeducação da marcha de pacientes hemiparéticos com a combinação dessas técnicas fisioterapêuticas. Sendo assim, o objetivo deste estudo é analisar os efeitos de um programa de intervenção fisioterapêutica neurofuncional combinando as técnicas de treino por tarefas orientadas e treino de marcha para trás sobre a locomoção de pacientes hemiparéticos após AVE.

\section{Material e métodos}

Este é um estudo de eficácia do tipo série de casos, no qual o sujeito é seu próprio controle e são feitas múltiplas avaliações no tempo, por um avaliador cego ao objetivo do estudo e que não estava envolvido nos atendimentos fisioterápicos. O estudo foi aprovado pelo Comitê de Ética em Pesquisa da Universidade Federal de Sergipe (UFS) com o parecer no 2.897.507, CAAE: 96441417.0 .0000 .5546 . Todos os participantes assinaram o termo de consentimento livre e esclarecido. A pesquisa foi desenvolvida na clínica escola do curso de fisioterapia da UFS Campus Professor Antônio Garcia Filho e no Centro de Especialidades Médicas (CEM), ambos da cidade de Lagarto/SE no ano de 2017 e 2018.

Participaram da pesquisa indivíduos com diagnóstico de AVE de ambos sexos, recrutados da Clínica Escola de Fisioterapia da UFS-Lagarto e do CEM- Lagarto, que manifestaram desejo de participar do estudo. Os critérios de inclusão foram: ter o diagnóstico de AVE, andarem sem risco de quedas, com pontuação na escala de Berg acima de 40 [32,33], demonstrar capacidade de entender instruções e seguir comandos simples, apresentando 
pontuação igual ou maior que 25 (ou 13 pontos no caso de analfabetos e 18 para baixa ou média escolaridade) no Mini Exame do Estado Mental (MEEM) [34,35]. Os critérios de exclusão foram: apresentar comorbidades ou incapacidades funcionais que fossem sequelas além do AVE ou que impedisse o treinamento da marcha, ou ainda, se apresentassem condição de saúde na qual o exercício/atividade física fosse contraindicado.

Foram utilizados nove instrumentos, sendo dois deles usados como triagem e empregados para avaliar os critérios de inclusão. As avaliações de triagem ou seleção de participantes foram a Escala de Equilíbrio de Berg e o Mini Exame do Estado Mental (MEEM).

A Escala de Equilíbrio de Berg analisa o controle postural estável e antecipatório e possui pontuação máxima de 56 pontos, na qual quanto maior a pontuação melhor o desempenho, sendo escores maiores que 40 indicativos de marcha independente [27], só foram incluídos no estudo participantes com Berg > 40. O MEEM foi utilizado para mensurar as funções cognitivas, e possui escore total de 30 pontos. Há também pontos de corte baseados no nível de escolaridade, sendo 13 pontos para analfabetos; 18 pontos para indivíduos com baixa ou média escolaridade; e 26 pontos para aqueles com alto nível de escolaridade [32,33]. Participaram do estudo apenas os voluntários acima do ponto de corte considerando o nível de escolaridade.

Instrumentos de classificação clínica associados ao desempenho da marcha

Com o intuito de classificar clinicamente os participantes, já que sua condição física pode influenciar diretamente 0 desfecho primário, foram selecionadas quatro avaliações. A Classificação Funcional da Marcha Modificada (CFMM) classifica o desempenho da locomoção, apresenta pontuação máxima de 5 e mínima de 0 , e quanto maior a pontuação, melhor 0 desempenho [36]. Também foi utilizada a Escala de Fugl-Meyer, que avalia a condição do paciente em cinco domínios, sendo eles função motora, sensibilidade, amplitude de movimento e dor para membros inferiores e superiores, e equilíbrio postural [37]. No entanto, para o presente estudo, tivemos o interesse de utilizar a escala Fugl-Meyer dos MMII completa (inclui sensibilidade, dor e amplitude de movimento dos MMII) tendo um valor máximo de 86 pontos. Muitos estudos apenas utilizam a parte funcional da escala Fugl-Meyer que para os membros inferiores tem um valor de 34 pontos [37,38]. Como existem evidência que os membros superiores podem influenciar no desempenho funcional da marcha [39-41], foi utilizado o Box and Blocks test (BBT) que avalia a destreza e habilidade manual unilateral [39]. A hipertonia do tipo espástica pode causar sinergias patológicas na marcha de pessoas com sequelas de AVE [42]. Assim, foi utilizado o Índex Composto de Espasticidade (ICE), que verifica os três componentes básicos da espasticidade: a excitabilidade fásica do reflexo, a resistência ao estiramento muscular, e a presença de clônus, tendo sua pontuação variando entre 1 a 16, em que o valor do score é diretamente proporcional a severidade do quadro $[42,43]$.

\section{Instrumentos utilizados para investigar os desfechos primários ou efeito do tratamento}

Como desfechos primários, com a finalidade de quantificar as evoluções após tratamento foram aplicadas três avaliações. 1) A escala Fullerton Advanced Balance (FAB) que avaliou o equilíbrio funcional dos participantes, composta por 10 itens, tendo 40 como pontuação total. Resultados menores ou igual a 25 representam alto risco para quedas e indica necessidade de intervenção imediata [44]. 2) Índice de Marcha Dinâmica ou Dynamic Gait Index (DGI), que mede a capacidade do participante de modificar a marcha em resposta às exigências da tarefa funcional, composta por 8 itens pontuados de 0 a 3, onde 0 significa comprometimento grave e 3 sem comprometimento ou normal [45]. 3) O Timed Up and Go (TUG) avalia a mobilidade e equilíbrio funcional ao medir o tempo que o indivíduo leva para realizar manobras funcionais como levantar, caminhar, dar uma volta e sentar em uma distância de 6 metros [46]. Por segurança, durante as avaliações e tratamento os participantes tiveram os sinais vitais monitorados.

\section{Intervenção}

O presente estudo ocorreu na Clínica Escola de Fisioterapia da Universidade Federal de Sergipe/Campus Lagarto e no Centro de Fisioterapia do Município de Lagarto. Este contou com um grupo de fisioterapeutas que foram capacitados para treinar um único indivíduo por atendimento. O protocolo de intervenção foi desenvolvido com base na literatura existente sobre 
tarefas orientadas [14-23] e no treino com marcha para trás no solo [24-31]. Tinha a frequência de 2 vezes por semana, com duração de 60 minutos (1h) por atendimento, total de 10 sessões (1 mês e meio). Uma hora de treino era dividido da seguinte forma: 5 min de aquecimento, 25 min de marcha para trás, 25 min de tarefas orientadas e 5 min de desaquecimento. Dentro do bloco de atividades de marcha para trás poderiam ser feitas 5 atividades de acordo com a progressão do participante e no bloco de tarefas orientadas, poderiam ser feitas 4 atividades que também eram realizadas de acordo com a evolução do paciente durante os atendimentos.

Protocolo de intervenção das técnicas de tarefas orientadas e marcha para trás (cf Apêndice em PDF)

Foram feitas múltiplas avaliações, total de 6 que foram divididas nas fases A1, B, A2. A fase A1 consistiu em duas avaliações antes de começar o tratamento (com intervalo de 3-5 dias entre a primeira e a segunda) permitindo o cálculo de uma linha de base ou baseline. Durante (5 dia após início do tratamento) e imediatamente após o tratamento (10 ia ou último dia de tratamento), foram realizadas duas medidas, formando a fase B. A fase A2 consistiu em mais duas avaliações posteriores ao tratamento ( 15 e 30 dias após o término do tratamento).

Na metodologia por série de casos [47,48], é necessário no mínimo em cada fase duas avaliações. Essa quantidade fornece dois pontos de dados da mesma medida com variação, permitindo então análise estatística dos dados. Menos de dois pontos por medida não permite análise estatística em estudos cujo sujeito é controle dele mesmo e mais de dois pontos é arriscado a incluir o fator de aprendizagem das medidas. Os avaliadores eram cegos aos objetivos da pesquisa e não estavam envolvidos no tratamento. Uma terceira pessoa não envolvida com a avaliação ou tratamento tabulou os resultados das avaliações.

\section{Análise estatística}

Foram utilizados dois métodos estatísticos (Fig. 1):

A) Método visual da banda formada pela média e por dois desvios-padrão calculados na baseline: os scores que se encontram dentro da banda formada não possuem significância estatística (na imagem, os pontos 6 e 8), já os pontos que saem da banda para cima ou para baixo (na imagem, 10 e 12; e 4 respectivamente) significa que o tratamento surtiu efeito, seja ele positivo ou negativo, com $\mathrm{p}<0,05$ [49].

B) Método do cálculo do tamanho de efeito da terapia: baseado nas médias e desvios-padrão individuais e do grupo expressa a relevância clínica da diferença entre os scores obtidos antes, durante e após o tratamento [50-52].

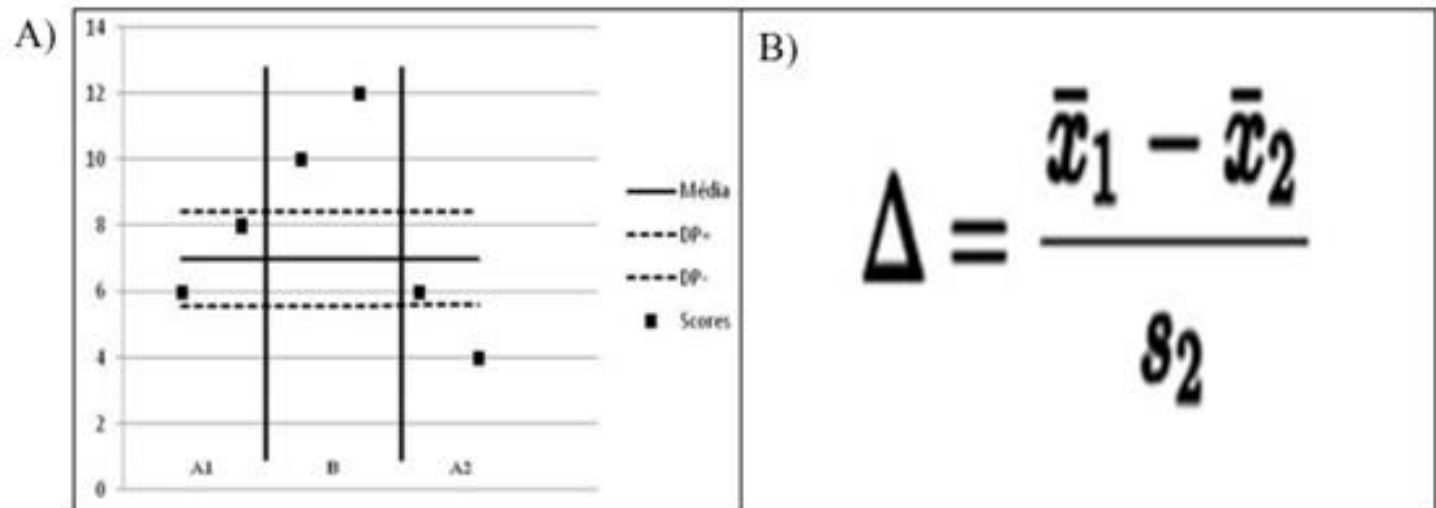

Figura 1 - A) Método visual da banda formada pela média e por dois desvios padrões. B) Fórmula do cálculo do tamanho de efeito da terapia.

\section{Resultados}

No período de agosto de 2017 a março de 2018, cinco pacientes com hemiparesia secundária ao AVE aceitaram participar deste estudo. Porém, somente quatro pacientes concluíram as dez sessões de tratamento. A desistência de um participante justificou-se em 
virtude de intercorrências relacionadas a problemas de saúde, que impossibilitou uma frequência adequada aos atendimentos.

A amostra consistiu em três participantes do sexo masculino e um do sexo feminino, com idade variante entre 58 e 75 anos, apresentando AVE tanto do tipo isquêmico como hemorrágico, a maioria possuindo mais de um episódio (Tabela I). Todos já estavam em alta hospitalar e estavam no período ambulatorial, já tinham feito tratamento fisioterapêutico, três ainda permaneciam em tratamento durante o período da pesquisa por motivos diversos (melhora funcional no membro superior ou atividades diversas, inclusive a marcha). Por motivos éticos, aqueles que continuavam em tratamento fisioterapêutico, durante a pesquisa, não foram impedidos de continuar o mesmo, porém, como o tratamento de rotina daqueles que continuaram também era realizado na clínica escola de Fisioterapia da UFS Lagarto, todo o tratamento passou a ser voltado para o membro superior e não coincidia em conteúdo e forma com o treino de tarefas orientadas e marcha para trás. Além desse cuidado, lembramos que este estudo adota a metodologia de análise de resultados de cada voluntário individualmente, com o efeito do tratamento comparado a uma baseline resultante de duas medidas antes do tratamento proposto por este estudo, ou seja, a evolução do voluntário é comparada com ele mesmo, ou com o estado funcional que ele se encontrava antes de começar o tratamento proposto por esse estudo. Consequentemente, o fato de termos apenas um voluntário que não fazia a fisioterapia convencional para membro superior não interfere nos resultados dos outros três voluntários.

Tabela I - Caracterização da amostra quanto a dados demográficos e instrumentos clínicos classificatórios.

\begin{tabular}{|c|c|c|c|c|c|c|c|c|c|}
\hline & \multirow[t]{2}{*}{ Sexo } & \multirow{2}{*}{$\begin{array}{l}\text { Lado + } \\
\text { Afetado }\end{array}$} & \multirow[t]{2}{*}{ Idade } & \multirow{2}{*}{$\begin{array}{l}\text { Número } \\
\text { de AVE }\end{array}$} & \multicolumn{2}{|l|}{ BBT } & \multirow[t]{2}{*}{ ICE } & \multirow{2}{*}{$\begin{array}{l}\text { Fugl- } \\
\text { Meyer } \\
\text { MMII } \\
\text { completa }\end{array}$} & \multirow[t]{2}{*}{ CFMM } \\
\hline & & & & & $D$ & $E$ & & & \\
\hline P1 & $M$ & $E$ & 66 & 1 & 49 & 53,5 & 4 & 50 & 4 \\
\hline P2 & $\mathrm{F}$ & $\mathrm{E}$ & 75 & $2+$ & 50,5 & 22,5 & 6 & 58 & 3 \\
\hline P3 & $M$ & $\mathrm{E}$ & 58 & $2+$ & 45,6 & 21 & 11 & 67 & 4 \\
\hline $\mathbf{P 4}^{\star}$ & $M$ & $\mathrm{D}$ & 64 & $2+$ & 20,5 & 35,5 & 7 & 66 & 2 \\
\hline
\end{tabular}

AVE = Acidente Vascular Encefálico, BBT = Box and Blocks Test; ICE = Index Composto de Espasticidade, CFMM = Classificação Funcional da Marcha Modificada. *P4 foi o único paciente que não continuou os atendimentos da fisioterapia convencional.

Em relação aos resultados obtidos a partir da análise da aplicação do instrumento $F A B$, inferiu-se que dois participantes responderam de forma positiva ao protocolo de intervenção e reteram essa melhora um mês após o tratamento (P1 e P2). Já P3, embora tenha obtido melhor desempenho na fase $B$, não conseguiu estendê-lo para a fase $A 2$ do tratamento. O participante P4 apresentou piora dos resultados no equilíbrio durante e imediatamente após o tratamento, atingindo scores mais baixos em $\mathrm{B}$ quando comparados com A1, mas voltou ao desempenho inicial na segunda medida de A2, ou seja, após 30 dias sem o tratamento (Figura 2).

A análise dos scores obtidos a partir da aplicação do instrumento DGI revelou aumento significativo da pontuação após o protocolo de intervenção, implicando em melhores scores dos participantes P2, P3 e P4 (Figura 2). Os três participantes obtiveram este resultado somente durante a fase $\mathrm{A}$ ou imediatamente após o tratamento, fase B. Não houve retenção dos efeitos do tratamento, como observado que os pontos na fase A2 (15 e 30 dias após tratamento) voltam para dentro da banda. Com exceção de P3 que na avaliação com 30 dias sem tratamento (segunda avaliação A2), teve uma queda significativa no seu score do DGI (Figura 3). 


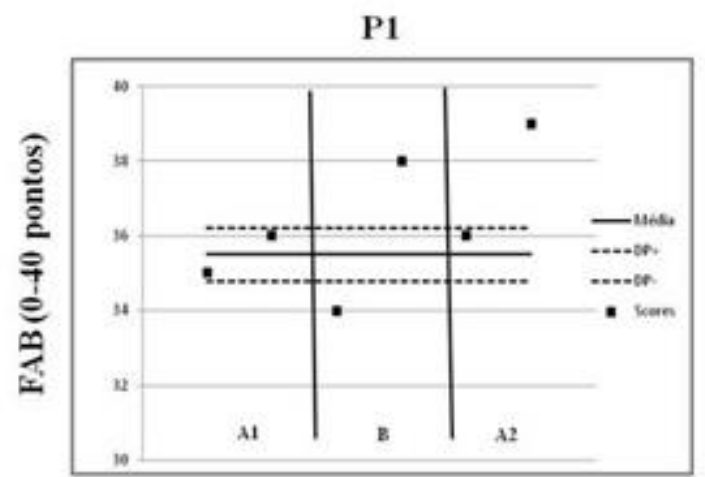

P3

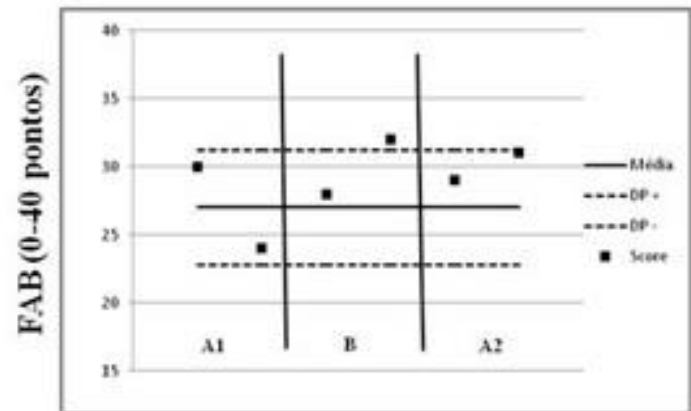

P2

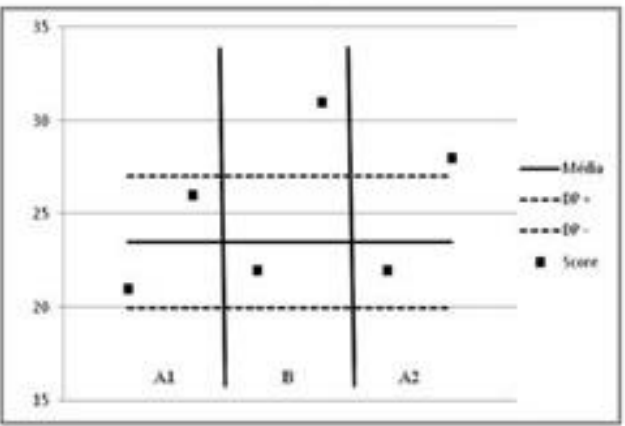

P4

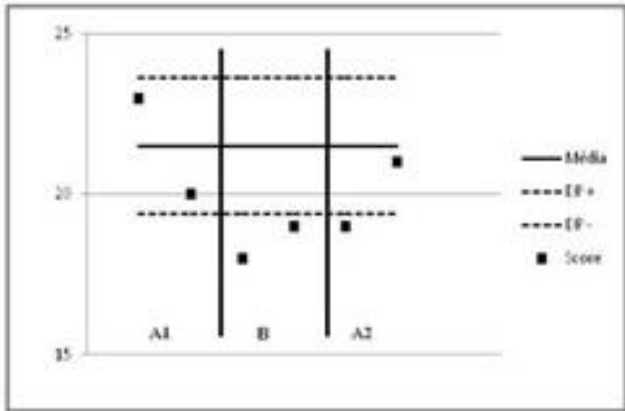

Figura 2 - Análise Teste FAB para cada participante com o método de banda composta pela média e 2 desvios padrões (DP) da baseline. Os pontos representam cada avaliação feita, quando um ponto sai da banda representa uma mudança significativa com nível alpha $p<0,05$. Fase $A 1=$ Avaliação antes do tratamento, fase $B=$ avaliação durante o tratamento e fase $A 2=$ após o tratamento.

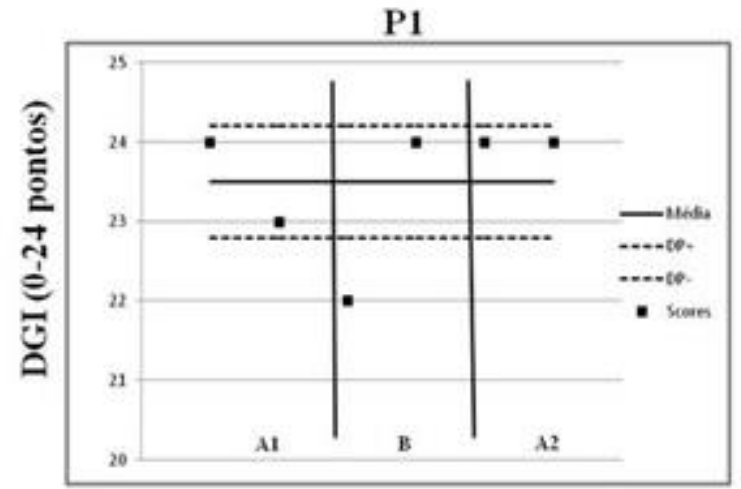

P3

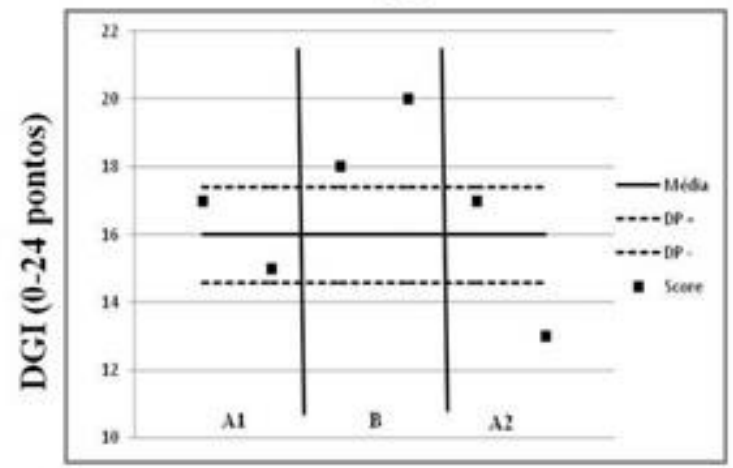

P2

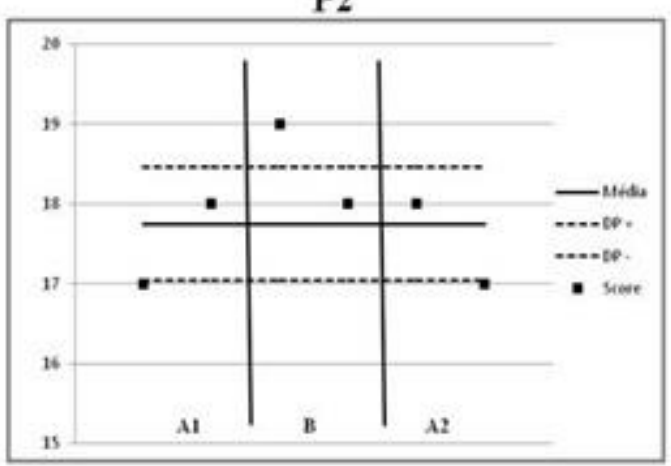

P4

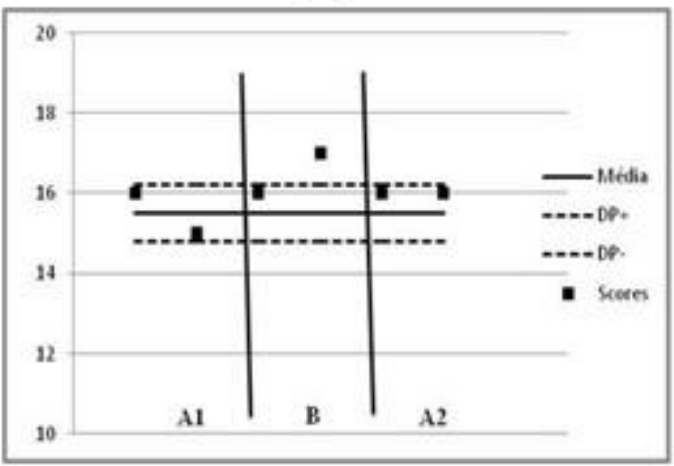

Figura 3 - Análise para cada participante no Teste DGI com o método da banda composto pela média e 2DP da baseline. Os pontos que saem da banda representam valores significativos $p<$ 
0,05 . Fase $A 1$, avaliação antes do tratamento, fase $B$ avaliação durante e imediatamente após 0 tratamento e fase $A 215$ e 30 dias após o tratamento.

$\mathrm{Na}$ avaliação do efeito do tratamento com o instrumento TUG foi observado que todos os participantes apresentaram desfecho positivo, com redução dos segundos necessários para realização completa do teste. Os participantes P1 e P2 demonstraram melhora significante na fase A2. Já o participante $\mathrm{P} 3$ apesar de ter melhorado na fase $B$, não conseguiu manter a melhora no TUG durante as medidas da fase A2 (15 dias e 30 dias após o tratamento). P4 apresentou melhora dos scores tanto na fase B como na $A 2$, esta última fase menos marcante que 0 anterior (Figura 4).

P1

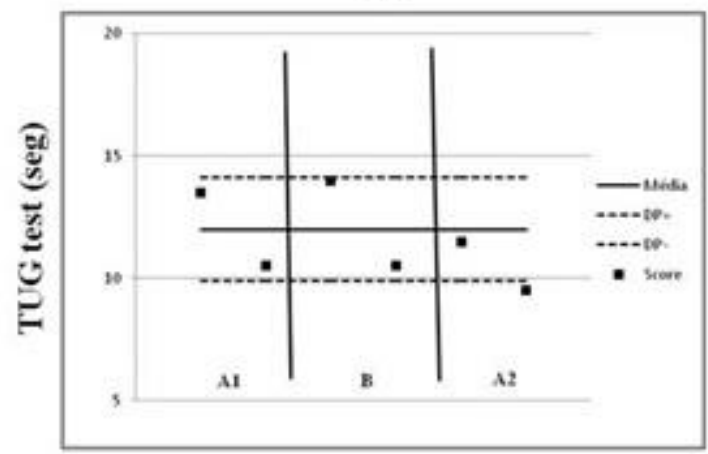

P3

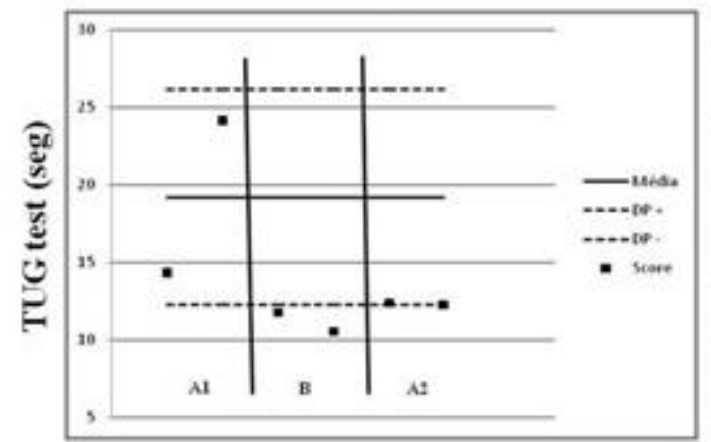

P2

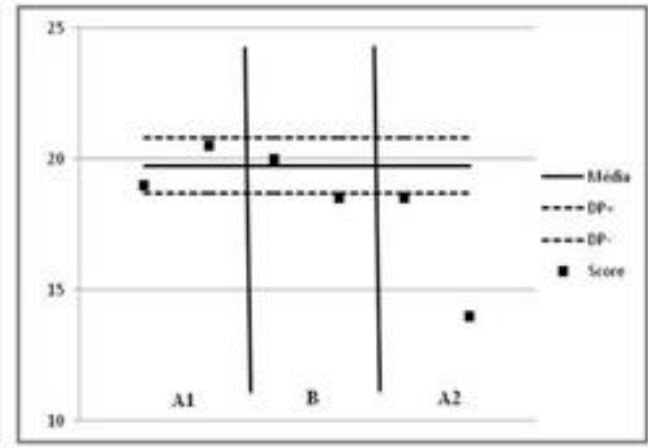

P4

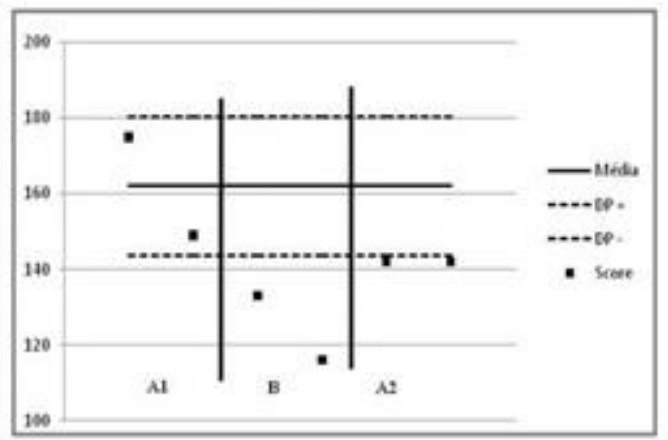

Figura 4 - Análise para cada participante no Teste TUG com o método da banda composta pela média e 2DP da baseline. Os pontos que saem da banda representam valores significativos $p<$ 0,05 . Fase $A 1$ Avaliação antes do tratamento, fase $B$ avaliação durante e imediatamente após 0 tratamento e fase A2 15 dias e 30 dias após o tratamento.

Os tamanhos do efeito da terapia estão representados na Tabela II, para cada paciente e nessa tabela foi calculado o tamanho do efeito para o grupo de pacientes. O tamanho do efeito da terapia foi calculado através da equação de Glass delta, para cada desfecho primário, baseado na diferença da média dos valores durante e imediatamente após o tratamento (fase $B$ ) e da média dos valores 15 dias e após 30 dias sem tratamento (A2), da média das medidas observadas na baseline.

Tabela II - Tamanho do efeito da terapia, para cada paciente voluntário e para o grupo.

\begin{tabular}{llllllll}
\hline Pacientes & \multicolumn{3}{c}{ FAB } & \multicolumn{3}{c}{ DGI } & TUG \\
\hline Participantes & $\begin{array}{l}\text { Fases } \\
\text { tratamento }\end{array}$ & B & A2 & B & A2 & B & A2 \\
\hline P1 & & 1,41 & 3,52 & 0 & 0 & 0 & 0,71 \\
P2 & & 0,85 & 0,42 & 1,06 & $-0,35$ & 0,47 & 3,30 \\
P3 & & 0,71 & 0,71 & 2,13 & $-0,71$ & 1,16 & 0,99 \\
P4 & & $-1,42$ & $-0,71$ & 0,70 & 0 & 2,04 & 1,09 \\
Grupo* & & 0,17 & 0,80 & 0,21 & 0,74 & 0,16 & 0,65 \\
\hline
\end{tabular}


Cálculo do tamanho do efeito usando a média das duas medidas de cada fase (B) e (A2). Sendo utilizados como valores de referência: $0=$ sem efeito, $<0,19$ insignificante, 0,20 a 0,49 pequeno, 0,50 a 0,79 médio, 0,80 a 1,29 grande $>1,30$ muito grande [52]. Em cinza e em negrito estão os efeitos positivos da terapia experimental. Números do tamanho do efeito precedidos do sinal negativo e em itálico significam que o participante deteriorou ou diminuiu o escore durante ou após o tratamento em relação ao que obteve antes do tratamento ou baseline. * Tamanho do efeito considerando o grupo, a média do desempenho dos quatro participantes.

O resumo dos resultados obtidos a partir do efeito durante e imediatamente após a terapia experimental na fase B, referentes aos instrumentos de desfecho primário (Fullerton, DGI e TUG) estão na Tabela III.

Tabela III - Resumo dos resultados obtidos sobre o efeito da terapia, calculado a partir da média das duas avaliações durante e imediatamente após o tratamento, fase $B$.

\begin{tabular}{llll} 
Participantes & FAB & DGI & TUG \\
\hline P1 & + & 0 & 0 \\
P2 & + & + & + \\
P3 & + & + & + \\
P4 & - & + & +
\end{tabular}

"+" significa que o tratamento teve um efeito positivo no score do instrumento utilizado quando comparado a fase baseline (A1), " - "significa que houve um efeito negativo no score do instrumento utilizado, e "0" significa que não surtiu nenhum efeito.

Discussão

Este estudo analisou os efeitos de um programa de intervenção fisioterapêutica de tarefas orientadas e treino de marcha para trás no solo, sobre a locomoção de pacientes hemiparéticos devido a acidente vascular encefálico (AVE). Para isso foram utilizados os instrumentos que avaliaram equilíbrio dinâmico, marcha funcional e mobilidade: FAB, DGI e TUG, respectivamente. Participaram do estudo quatro voluntários, em todos os quatro participantes após o tratamento foi observada melhora em aspectos da marcha.

Para participar deste estudo, os participantes tinham que ter um escore maior que 40 na escala de equilíbrio de Berg, o que significa que todos os quatro participantes tinham um equilíbrio satisfatório e seguro para o treino locomotor com marcha para trás [24,32,33]. $\mathrm{Na}$ avaliação do efeito do programa fisioterapêutico de tarefas orientadas e marcha para trás sobre o equilíbrio dinâmico, utilizou-se o instrumento $F A B$, pois esse possui itens que solicitam um equilíbrio mais avançado que os itens da escala de Berg, englobando aspectos mais dinâmicos do equilíbrio [44]. Três participantes aumentaram o score do FAB durante e imediatamente após o tratamento (fase B) (P1, P2 e P3), exceto o participante 4 (P4) que na fase B piorou os escores do $F A B$ mostrando uma deterioração no equilíbrio dinâmico, logo após o tratamento. $O$ participante 4 (P4) na CFMM (Classificação Funcional da Marcha Modificada) foi classificado com score 2 de 5, possuindo apenas uma marcha domiciliar [36].

Apesar de ter um score maior que 40 na escala de Berg, o treino com tarefas orientadas e marcha para trás foi um desafio para ele, ajustes posturais e tarefas novas tinham que ser aprendidas e quando aprendidas deveriam ser progredidas (protocolo detalhado da intervenção no Apêndice). Após o tratamento imediato, essa dificuldade de P4 ficou evidente nos escores da escala de equilíbrio avançada de Fullerton (FAB) na fase $B$, e na fase $A 2$, com 15 e 30 dias sem tratamento, onde nessa última fase, os escores começaram a voltar a ter os números parecidos com o da baseline. $O$ participante 4 também foi o único que não continuou o tratamento fisioterapêutico convencional para os MMSS, mas pelo seu score do Box and Blocks, Índex Composto de Espasticidade (ICE) e Fulg-Meyer MMII completo (Tabela I) sua função motora geral não estava tão diferente dos outros três participantes. A principal diferença do $\mathrm{P} 4$ é que ele teve dois $A V E$ e os dois foram do lado esquerdo do encéfalo, sendo o único com o lado direito hemiparético.

Existem evidências na literatura que o planejamento das atividades motoras é mais dependente do hemisfério cerebral esquerdo, logo, a aprendizagem motora nos pacientes com lesão no hemisfério cerebral esquerdo são mais prejudicadas [53]. O treino com tarefas orientadas e treino de marcha para trás seguiu um protocolo padronizado para os quatro participantes neste estudo (Apêndice), clinicamente o treino por tarefas orientadas é individualizado e baseado nas dificuldades funcionais de cada indivíduo [17]. Dessa forma, o P4 teve maiores dificuldades de adaptação e aprendizagem, diferindo dos pacientes P1, P2 e P3 
que possuem acometimento no hemisfério direito e o protocolo de intervenção não foi adaptado para essa especialidade que P4 tinha de aprendizagem motora.

$\mathrm{O}$ instrumento DGI mede a marcha durante tarefas funcionais, o programa de treino fisioterapêutico utilizado neste estudo não teve efeito para P1 na avaliação com esse instrumento. Isto pode ser atribuído ao fato de o participante ter um bom nível funcional quando comparado aos outros; percebido através do bom desempenho bilateral no instrumento BBT, ausência de espasticidade e CFMM = 4, com marcha comunitária independente; diferentemente de P2, P3 e P4 que possuíam maior espasticidade no lado hemiparético, diferença importante entre os desempenhos dos MMSS no BBT e classificações mais severas na CFMM (Tabela I).

Apesar de haver uma tendência de indivíduos com AVE classificados como mais leves se recuperarem bem, a sua evolução durante o tratamento através de escalas clínicas padronizadas podem ser prejudicadas por um efeito de teto da escala, onde os participantes que são graves ou na faixa de gravidade média acabam por ter suas mudanças melhores detectadas pelas escalas. Embora seja um instrumento confiável, a escala DGI possui características que diminuem sua sensibilidade clínica para detectar as alterações de equilíbrio durante a locomoção, o que por consequência interfere nos scores gerais e pode permitir interpretações que não condizem com a repercussão clínica [45].

Exemplo é o item 5 que solicita que o indivíduo ande, vire-se o mais rápido que puder na direção contrária e pare de frente a seu ponto de partida. Na classificação considerada normal, o paciente deve girar o corpo em até 3 segundos sem perder o equilíbrio. Porém, durante a execução do teste, pode acontecer que o paciente gire o corpo rapidamente em até 3 segundos, mas apresentando instabilidade após a parada; no entanto, não há opção de graduação para este resultado, o que pode tendenciar o avaliador a considerar prioritariamente o tempo e desconsiderar a qualidade de execução da tarefa [45]. Levando em conta este exemplo, um mesmo indivíduo poderia realizar a tarefa em 3 segundos e com desequilíbrio na fase $A 1$ e na fase $B$ ou $A 2$ realizar em 3 segundos e sem instabilidade; ambos os desempenhos receberiam a mesma pontuação, não sendo detectada a evolução clínica de melhora na instabilidade. Devese sempre ter cuidado para não confundir significância estatística com significância clínica. Apesar de o valor do $p$ mostrar a probabilidade de se obter uma estatística significativa, ele não é capaz de informar sobre a importância clínica ou prática dos resultados obtidos $[47,50]$.

Quanto a retenção do tratamento, exposta na fase A2, nenhum dos participantes obtiveram êxito na avaliação com a escala DGI: Um estudo com treino de marcha para trás em esteira rolante [54] também utilizou o DGl como desfecho e não obteve sucesso na fase de retenção do tratamento. Na discussão os autores também mencionam o efeito teto que esse instrumento pode apresentar.

No TUG, todos os participantes tiveram benefícios com a terapia por tarefas orientadas e treino de marcha para trás no solo. O Timed up and go (TUG) é uma medida de equilíbrio e mobilidade funcional, em que o paciente deve se levantar de uma cadeira andar $3 \mathrm{~m}$, retornar de volta os $3 \mathrm{~m}$ e sentar de novo na cadeira. A distância percorrida do TUG é fixa, total $6 \mathrm{~m}$, o que se mede no TUG é o tempo, logo indiretamente o TUG informa a velocidade da marcha, em nenhum momento, no TUG original, a qualidade do movimento é avaliada. $O$ efeito esperado da terapia no TUG é uma diminuição do tempo para executar a tarefa [46]. Vários estudos que utilizaram o treino por tarefas orientadas utilizaram o TUG como desfecho primário $[55,56]$ e mostraram resultados benéficos após a terapia. Apenas dois estudos recentes, Moon and Bae [57] e DeMark et al. [58] realizaram como intervenção o treino a marcha para trás em pacientes com sequelas de AVE, e utilizaram o TUG como desfecho. Assim como este estudo, os dois estudos mencionados $[57,58]$ reportaram resultados benéficos com o TUG após o programa fisioterapêutico com marcha para trás, mas os protocolos de intervenção utilizados em ambos estudos são diferentes do apresentado neste estudo. Mae and Bae [57] utilizaram o treino em que os participantes apenas deveriam efetuar a observação da marcha para trás, já DeMark et al. [58] é um dos primeiros estudos semelhantes ao nosso, no sentido que utilizou o treino de marcha para trás no solo, mas não utilizou um protocolo de treino por tarefas orientadas.

De acordo com os resultados encontrados neste estudo, os indivíduos hemiparéticos após AVE apresentaram de forma geral melhora do equilíbrio funcional, marcha e mobilidade durante a aplicação do protocolo de treino por tarefas orientadas e marcha para trás. Esses resultados em parte corroboram alguns estudos que aplicaram o treino por tarefas orientadas ou o treino de marcha para trás [54-58]. Porém, este é o primeiro ensaio clínico que investiga o uso de um programa por treino por tarefas orientadas e treino de marcha para trás juntos.

Apesar de ter uma pequena amostra, o desenho metodológico deste estudo foi robusto por realizar uma análise individual ou série de casos de maneira controlada e recomendada por 
ensaios clínicos desse tipo [59]. Podemos atribuir a dificuldade de recrutamento de voluntários aos seguintes fatores: 1) Transporte, os participantes selecionados não tinham condições de se deslocar e também não tinham suporte do setor público; 2) Para participar o participante deveria ter um score maior que 40 pontos na Escala de Equilíbrio de Berg, pois o participante precisava andar para poder fazer parte do estudo. Visto que a seleção dos participantes é feita no setor público, e grande parte dos acometidos por AVE possuem sequelas motoras graves, foi difícil achar participantes com escores iguais ou maiores que 40 pontos na escala de Equilíbrio de Berg.

Apesar das limitações em termo de generalização dos resultados pela pequena amostra, os resultados deste estudo podem contribuir de forma significante para a Fisioterapia Neurofuncional, pois a metodologia por análise individual de voluntários (Single Subject Experimental Designs) está cada vez mais tendo importância na aplicação clínica das evidências científicas ou práticas baseadas em evidência [60]. Este estudo faz parte de um projeto de estratégias fisioterapêuticas neurofuncionais para aumentar a habilidade de pacientes com sequelas de AVE de se locomoverem na comunidade, visto que na realidade as tarefas funcionais da marcha são mais desafiadoras que na clínica e no laboratório de pesquisa [61]. Esperamos que os resultados deste estudo incentivem o desenvolvimento de estudos semelhantes com maior número de participantes, utilizando o programa fisioterapêutico de tarefas orientadas e treino com marcha para trás.

\section{Conclusão}

Este é o primeiro estudo que investigou o efeito do programa fisioterapêutico de tarefas orientadas e treino de marcha para trás juntos na locomoção de pacientes após AVE. Os resultados indicam que os participantes foram capazes de melhorar o equilíbrio, mobilidade, locomoção e resposta as demandas funcionais da marcha imediatamente após o tratamento e mantiveram alguns aspectos desses benefícios um mês após o tratamento. Por se tratar de um estudo de série de casos é recomendado ensaios clínicos com uma amostra maior para verificar a eficácia dos resultados.

\section{Referências}

1. Billinger SA, Arena R, Bernhardt J, Eng JJ, Franklin BA, Johnson CM, Shaughnessy M. Physical activity and exercise recommendations for stroke survivors: a statement for healthcare professionals from the American Heart Association/American Stroke Association. Stroke 2014;45(8):2532-53. https://doi.org/10.1161/STR.0000000000000022

2. Joseph C, Rhoda A. Activity limitations and factors influencing functional outcome of patients with stroke following rehabilitation at a specialized facility in the Western Cape. Afr Health Sci 2013;3(3):646-54. https://doi.org/10.4314/ahs.v13i3.18

3. Reis RD, Pereira EC, Pereira MIM, Soane AMNC, Silva JV. Significados, para os familiares, de conviver com um idoso com sequelas de acidente vascular cerebreal (AVC). Interface (Botucatu) 2016;21(62). https://doi.org/10.1590/1807-57622016.0206

4. Gomes MJAR. Vidas após um acidente vascular cerebral: efeitos individuais, familiares e sociais [Tese]. Minho: Universidade do Minho; 2012.

5. Ministério da Saúde (BR). Datasus. Banco de dados do sistema único de saúde [internet]; 2016 [citado 06 jul 2016]. Disponível em: http://www.datasus.gov.br

6. Dyer JO, Maupas E, Andrade MS, Bourbonnais D, Forget R. Abnormal coactivation of knee and ankle extensors is related to changes in heteronymous spinal pathways after stroke. J Neuroeng Rehabil 2011;8(1):41. https://doi.org/10.1186/1743-0003-8-41

7. Connell LA, Lincoln NB, Radford KA. Somato sensory impairment after stroke: frequency of different deficits and their recovery. Clin Rehabil 2008;2(8):758-67. https://doi.org/10.1177/0269215508090674

8. Dyer JO, Maupas E, Andrade MS, Bourbonnais D, Fleury J, Forget R. Transmission in heteronymous spinal pathways is modified after stroke and related to motor incoordination. PloS one 2009;4(1):e4123.

9. Moreira RP, Araujo TL, Cavalcante TF, Guedes NG, Costa AG, Lopes MV. Validation of the mobility nursing outcome in stroke survivors. Int J of Nurs Knowl 2013;24(3):157-62. https://doi.org/10.1111/j.2047-3095.2013.01245.x 
10. Lord SE, Rochester L, Weatherall M. Community ambulation after stroke: how important and obtainable is it and what measures appear predictive? Arch Phys Med Rehabil 2004;85(2):234-9. https://doi.org/ 10.1016/j.apmr.2003.05.002

11. Corrêa FI, Soares F, Andrade DV, Gondo RM, Peres JA, Fernandes AO, et al. Atividade muscular durante a marcha após acidente vascular encefálico. Arq Neuropsiquiatr 2005;63(3-B):847-51. https://doi.org/10.1590/S0004282X2005000500024

12. van Duijnhoven HJ, Geurts AC. Falls in individuals with stroke. J Rehabil Res Dev 2008;45(8):1195-213. https://doi.org/10.1682/JRRD.2007.09.0145

13. Seung-Hun An MS, Yun-Mi Lee MS, Kyung-Hee Y. Effectiveness of gait training using an electromechanical gait trainer combined with simultaneous functional electrical stimulation in chronic stroke patients. J Kor Soc Phys Ther 2008;20(1):41-7.

14. Eich $\mathrm{HJ}$, Mach H, Werner $\mathrm{C}$, Hesse S. Aerobic treadmill plus Bobath walking training improves walking in subacute stroke: a randomized controlled trial. Clinical Rehabil 2004;18:640-51. https://doi.org/10.1191/0269215504cr779oa

15. Bowden MG, Behrman AL, Neptune RR, Gregory CM, Kautz SA. Locomotor rehabilitation of individuals with chronic stroke: difference between responders and non responders. Arch Phys Med Rehabil 2013;94(5):856-62. https://doi.org/10.1016/j.apmr.2012.11.032

16. Wevers L, Van de port I, Vermue M, Mead G, Kwakkel G. Effects of task- oriented circuit class training on walking competency after stroke: a systematic review. Stroke 2009;40(7):2450-9.

17. Shumway-Cook A, Woollacott MH. Controle motor: teorias e aplicações práticas. Porto Alegre: Manole; 2003.

18. Schmidt R, Lee T. Aprendizagem e performance motora. 5 ed. Porto Alegre: ArtMed; 2016.

19. Schneiberg S, Miranda CB, Souza VTC, Paiva SV, Barroso-Carregosa E, Reis AF. Fisioterapia neurofuncional por tarefas orientadas em adultos pós acidente vascular encefálico: avaliação e prática. In: Sordi C, César C, Paranhos LP, eds. Coletâneas em Saúde. 1ed. São José dos Pinhais: Plena; 2016;5:105-17.

20. Chung SH, Kim JH, Yong SY, Lee YH, Park JM, Kim SH, Lee HC. Effect of task-specific lower extremity training on cognitive and gait function in stroke patients: a prospective randomized controlled trial. Annals of Rehabilitation Medicine 2019;43(1):1. https://doi.org/10.5535/arm.2019.43.1.1

21. Scianni A, Salmela T, Ada L. Effect of strengthening exercise in addition to task specific gait training after stroke: a randomised trial. Int J Stroke 2010;5(4):329-35. https://doi.org/10.1111//.1747-4949.2010.00449.x

22. Ferreira MJG. Efeitos de um programa baseado em tarefas orientadas na competência de marcha em indivíduos com acidente vascular cerebral [Tese]. 2012. Lisboa: Instituto Politécnico de Lisboa, Escola Superior de Tecnologia da Saúde de Lisboa; 2012. 82 f.

23. Blennerhassett J, Dite W. Additional task-related practice improve mobility and upper limb function early after stroke: a randomized controlled trial. Aust J Physiother 2004;50(4):219-24. https://doi.org/10.1016/s0004-9514(14)60111-2

24. Thomas MA, Fast A. One step forward and two steps back: the dangers of walking backwards in therapy. Am J Phys Medicine Rehabil 2000;79(5):459-61. https://doi.org/10.1097/00002060-200009000-00011

25. Yang YR, Yen JG, Wang RY, Yen LL, Lieu FK. Gait outcomes after additional backward walking training in patients with stroke: a randomized controlled trial. Clin Rehabil 2005;19(3):264-73. https://doi.org/10.1191/0269215505cr860oa

26. Hoogkamer W, Meyns P, Duysens J. Steps forward in understanding backward gait: from basic circuits to rehabilitation. Exerc Sport Sci Rev 2014;42(1):23-9. https://doi.org/10.1249/JES.0000000000000000

27. Nadeau S, Amblard B, Mesure S, Bourbonnais D. Head and trunk stabilization strategies during forward and backward walking in healthy adults. Gait Posture 2003;18(3):134-42. https://doi.org/10.1016/s0966-6362(02)00070-x

28. Kim HH, Shim JM. Comparison of forward and backward walking trainings on gait pattern in adults. Indian J Sci Technol 2016;9(43):1-5. https://doi.org/ $\underline{10.1589 / \text { jpts.29.187 }}$ 
29. Michaelsen, SM, Ovando AC, Romaguera F, Ada L. Effect of backward walking treadmill training on walking capacity after stroke: a randomized clinical trial. Int $\mathrm{J}$ Stroke 2014;9(4):529-32. https://doi.org/10.1111/ijs.12255

30. Rose DK, De Mark L, Fox EJ, Clark DJ, Wludyka P. A backward walking training program to improve balance and mobility in acute stroke: a pilot randomized controlled trial. J Neurol Phys Ther 2018;42(1):12-21. https://doi.org/10.1097/NPT.0000000000000210

31. Kim CY, Lee JS, Kim HD. Comparison of the effect of lateral and backward walking training on walking function in patients with poststroke hemiplegia: a pilot randomized controlled trial. Am J Phys Med Rehabil 2017;96(2):61-7. https://doi.org/10.1097/PHM.0000000000000541

32. Berg K, Maki B, Williams J. Clinical and laboratory measures of postural balance in an elderly population. Arch Phys Med Rehabil 1992;73:1073-80.

33. Ashburn A, Hyndman D, Pickering R., Yardley L, Harris S. Predicting people with stroke at risk of falls. Age Ageing 2008;37(3):270-6. https://doi.org/10.1093/ageing/afn066

34. Melo DM, Barbosa AJG. O uso do Mini-Exame do Estado Mental em pesquisas com idosos no Brasil: uma revisão sistemática. Cienc Saúde Coletiva 2015;20(12):3865-76. https://doi.org/10.1590/1413-812320152012.06032015

35. Folstein MF, Folstein SE, McHugh PR. "Mini-Mental State", a practical method for grading the cognitive state of patients for the clinician. J Psychiatr Res 1975;12:189-98. https://doi.org/10.1016/0022-3956(75)90026-6

36. Viosca E, Martinez JL, Almagro PL, Gracia A, Gonzáles C. Proposal and validation of a new functional ambulation classification scale for clinical use. Arch Phys Med Rehabil 2005;86(6):1234-8.

37. Fugl-Meyer AR, Jaasko L, Leyman I, Olsson S, Steglind S. The post-stroke hemiplegic patient. 1. a method for evaluation of physical performance. Scand J Rehabil Med 1975;7:13-31.

38. Padovani $C$ et al. Aplicação das escalas Fugl-Meyer Assessment (FMA) e Wolf Motor Function Test (WMFT) na recuperação funcional do membro superior em pacientes após acidente vascular encefálico crônico: revisão de literatura. Acta Fisiatr 2013;20(1):42-9.

39. Cavaco NS, Alouche SR. Instrumentos de avaliação da função de membros superiores após acidente vascular encefálico: uma revisão sistemática. Fisioter Pesqui 2010;17(2):178-83.

40. Mazzei LG, Bellinassi AF, Sousa PCA, Rodrigues CDA. A influência do membro superior na marcha de indivíduos com hemiparesia: revisão. Rev Fac Ciênc Méd Sorocaba 2016;18(2):75-8. https://doi.org/10.5327/Z1984-4840201623279

41. Ustinova KI, Feldman AG, Levin MF. Bilateral coupling facilitates recovery of rhythmical movements from perturbation in healthy and post-stroke subjects. Exp Brain Res 2013;227(2):263-74. https://doi.org/10.1007/s00221-013-3509-1

42. Levin MF, Hui-Chan MC. Relief of hemiparetic spasticity by TENS is associated with improvement in reflex and voluntary motor functions. Electroencephalogr Clin Neurophysiol 1992;85(2):131-42. https://doi.org/10.1016/0168-5597(92)90079-q

43. Almeida RR, Fontes VA, Knault Melo S, Knault LA, Schneiberg S. Tradução e validação da escala "composite spasticity index" em pacientes com sequela de acidente vascular encefálico. Anais V Cobrafin, Associação Brasileira de Fisioterapia Neurofuncional.

44. Klein PJ, Fiedler RC, Rose DJ. Rasch analysis of the Fullerton advanced balance (FAB). Physiother Can 2011;63(1):115-25. https://doi.org/10.3138/ptc.2009-51

45. De Castro SM, Perracini MR, Ganança FF. Versão brasileira do Dynamic Gait Index. Rev Bras Otorrinolaringol 2006;72(6):817-25.

46. Paula FL, Júnior EDA, Prata H. Teste "Timed Up and Go": uma comparação entre valores obtidos em ambiente fechado e aberto. Fisioter Mov 2007;(20)4:143-8.

47. Backman CL, Harris SR, Chisholm JAM, Monette AD. Single-subject research in rehabilitation: a review of studies using $A B$, withdrawal, multiple baseline, and alternating treatments designs. Arch Phys Med Rehabil 1997;78(10):1145-53. https://doi.org/10.1016/s0003-9993(97)90142-8

48. Zhan S, Ottenbacher KJ. Single subject research designs for disability research. Disability and Rehabilitation 2001;23(1):1-8. https://doi.org/10.1080/09638280150211202 
49. Bobrovitz CD, Ottenbacher KJ. Comparison of visual inspection and statistical analysis of single-subject data in rehabilitation research. Am J Phys Med Rehabil 1998;77(2):94102. https://doi.org/10.1097/00002060-199803000-00002

50. Espírito-Santo H, Daniel F. Calcular e apresentar tamanhos do efeito em trabalhos científicos (1): As limitações do $p<0,05$ na análise de diferenças de médias de dois grupos. Revista Portuguesa de Investigação Comportamental e Social 2015;1(1):3-16. https://doi.org/10.7342/ismt.rpics.2015.1.1.14

51. Olive ML, Smith BW. Effect size calculations and single subject designs. Educational Psychology 2005;25(2-3):313-24.

52. Kraemer HC, Kupfer DJ. Size of treatment effects and their importance to clinical research and practice. Biological Psychiatry 2006;59(11):990-6. https://doi.org/10.1016/i.biopsych.2005.09.014

53. Dimyan MA, Cohen LG. Neuroplasticity in the context of motor rehabilitation after stroke. Nat Rev Neurol 2011;7:76-85. https://doi.org/10.1038/nrneurol. 2010;200 68

54. Kim KH, Lee KB., Bae YH, Fong SS, Lee SM. Effects of progressive backward body weight supported treadmill training on gait ability in chronic stroke patients: A randomized controlled trial. Technology and Health Care 2017;25(5):867-76. https://doi.org/10.3233/THC-160720

55. Rensink M, Schuurmans M, Lindeman E, Hafsteinsdóttir T. Task-oriented training in rehabilitation after stroke: systematic review. J Adv Nurs 2009;65(4):737-54. https://doi.org/10.1111/j.1365-2648.2008.04925.X

56. Chung SH, Kim JH, Yong SY, Lee YH, Park JM., Kim SH, Lee HC. Effect of taskspecific lower extremity training on cognitive and gait function in stroke patients: a prospective randomized controlled trial. Ann Rehabil Med 2019;43(1):1. https://doi.org/10.5535/arm.2019.43.1.1

57. Moon Y, Bae Y. Backward walking observational training improves gait ability in patients with chronic stroke: randomised controlled pilot study. Int J Rehabil Res 2019;42(3):217-22. https://doi.org/10.1097/MRR.0000000000000352

58. DeMark L, Fox EJ, Spigel PM, Osborne J, Rose DK. Clinical application of backward walking training to improve walking function, balance, and fall-risk in acute stroke: a case series. Top Stroke Rehabil 2019;26(7):497-502. https://doi.org/10.1080/10749357.2019.1641011

59. Tate R, McDonald S, Perdices M, Togher L, Schultz R, Savage S. Rating the methodological quality of single-subject designs and n-of-1 trials: Introducing the SingleCase Experimental Design (SCED) Scale. Neuropsychol Rehabil 2008;18(4):385-401. https://doi.org/10.1080/09602010802009201

60. Krasny-Pacini A, Evans J. Single-case experimental designs to assess intervention effectiveness in rehabilitation: A practical guide. Ann Phys Rehabil Med 2018;61(3):16479. https://doi.org/10.1016/j.rehab.2017.12.002

61. Hawkins KA., Clark DJ, Balasubramanian CK, Fox EJ. Walking on uneven terrain in healthy adults and the implications for people after stroke. Neuro Rehabilitation 2017;41(4):765-74. https://doi.org/10.3233/NRE-1721 